\title{
Immunonkologisches Therapieprinzip beim Lungen- und Nierenzellkarzinom
}

\author{
Jürgen Wolf ${ }^{a}$ Viktor Grünwald \\ ${ }^{a}$ Centrum für Integrierte Onkologie an der Uniklinik Köln, Deutschland \\ ${ }^{b}$ Klinik für Hämatologie, Hämostaseologie, Onkologie und Stammzelltransplantation, Medizinische Hochschule Hannover, Deutschland
}

\section{Verbessertes molekulares Verständnis beim Lungenkarzinom}

Das Lungenkarzinom ist noch immer die weltweit führende Krebstodesursache [1]. Maßgebliche Faktoren für die ungünstige Prognose sind die frühe Metastasierung (nur $<20 \%$ der Patienten sind operabel) und die begrenzten systemischen Therapieoptionen [2,3]. Die seit Jahrzehnten beim nicht-kleinzelligen Bronchialkarzinom (NSCLC), dem häufigsten histologischen Subtyp, als Therapiestandard geltende platinbasierte Chemotherapie ist nur wenig wirksam - mit Ansprechraten von 20-30\% und einem medianen Überleben von ca. einem Jahr [4].

Eine substanzielle Verbesserung der systemischen Therapie gelang erst durch die Einführung genetisch-stratifizierter Therapieansätze. Insbesondere die Behandlung von Patienten mit aktivierenden Mutationen im epidermalen Wachstumsfaktorrezeptor (EGFR)-Gen (Häufigkeit ca. 12\% der Adenokarzinome) mit EGFR-gerichteten Tyrosinkinaseinhibitoren (TKI) und von Patienten mit Translokationen im ALK (anaplastic large cell lymphoma)-Gen (Häufigkeit ca. 3\% der Adenokarzinome) mit ALK-Inhibitoren ermöglichte signifikante Fortschritte in Tumoransprechen, -kontrolle und Überleben im Vergleich zur Chemotherapie [5-7].

Die Grenzen dieser neuen personalisierten Therapieansätze sind die fehlende Langzeitkontrolle, das regelmäßige Auftreten von Resistenzen und die Tatsache, dass diese Therapien aktuell nur für eine Minderheit der Patienten mit Lungenkrebs zur Verfügung stehen.

\section{Ansprechen auf Immuntherapie übertrifft die Erwartungen}

Traditionell galt das Lungenkarzinom über lange Zeit als «nicht immunogen»: Präklinische Modellvorstellungen ließen sich über viele Jahre hinweg nicht auf die klinische Situation übertragen. Umso überraschender wirkten die ersten Phase-IDaten, die beim American Society of Clinical Oncology (ASCO)-Meeting 2012 präsentiert wurden und das Potenzial des PD-1/PD-L1-Rezeptor/Liganden-Systems aufgezeigt haben $[8,9]$ : PD-1 und seine Liganden scheinen auch beim NSCLC eine zentrale Rolle einzunehmen, indem sie an der Aufrechterhaltung des tumorassoziierten immunsuppressiven Mikromilieus beteiligt sind (siehe auch «Immun-CheckpointMoleküle - negative Regulatoren der T-Zell-Antwort», Seite 4).

Diese Daten deuten darauf hin, dass bei einem Teil der Patienten mit einem therapierefraktären, metastasierten NSCLC erstmals eine im Vergleich zu den bisherigen Therapien länger anhaltende Tumorkontrolle erreicht werden kann. Möglicherweise bedingt die große genetische Heterogenität der NSCLC-Tumoren [10] aufgrund der damit korrelierten Veränderungen der Tumorantigenität eine besondere Vulnerabilität gegenüber einer Blockade des PD1-/PDL1-Signalwegs [11].

\section{Aktuelle Checkpoint-Inhibitor-Studien beim NSCLC}

Immunonkologische Therapieansätze gehörten zu den zentralen «Hot Topics» der diesjährigen Jahrestagung der ASCO. Auch eine offizielle ASCO-Pressekonferenz mit dem Titel «Progress in Immunotherapy» war diesem Thema gewidmet. Zahlreiche präsentierte Studien (Tab. 1) zu verschiedenen Anti-PD-1-Inhibitoren schlossen sowohl therapienaive als auch mehrfach vorbehandelte Patienten ein und evaluierten die Substanzen in Monotherapie oder im Rahmen verschiedener Kombinationsregime. Die Arbeitsgruppe von Brahmer et al. stellte aktualisierte Daten aus der initial beim ASCO 2012

\begin{tabular}{ll}
\hline KARGER & (1) 2014 S. Karger GmbH, Freiburg \\
2296-5270/14/3717-0010\$39.50/0 \\
$\begin{array}{l}\text { Fax +497614520714 } \\
\text { Information@Karger.com } \\
\text { www.karger.com }\end{array}$ & $\begin{array}{l}\text { Accessible online at: } \\
\text { www.karger.com/ort }\end{array}$
\end{tabular}


Tab. 1. ASCO-Highlights 2014 zum Anti-PD-1/PD-L1-Therapieprinzip beim NSCLC

\begin{tabular}{|c|c|c|}
\hline Autoren, Abstract-Nummer & Studienphase & Therapie \\
\hline Brahmer JR et al., 8112 & I & $\begin{array}{l}\text { BMS-936558 (anti-PD-1, ONO-4538) beim vorbehandelten, fortgeschrittenen } \\
\text { NSCLC: Überlebensdaten/Daten zur klinischen Aktivität/Subgruppenanalyse }\end{array}$ \\
\hline Antonia SJ et al., 8113 & I & $\begin{array}{l}\text { BMS-936558 (anti-PD-1, ONO-4538) plus platinbasierte Doublette beim fortge- } \\
\text { schrittenen NSCLC (IIIB/IV): Daten zum 1-Jahres-Überleben, Sicherheit }\end{array}$ \\
\hline Antonia SJ et al., 8023 & I & $\begin{array}{l}\text { BMS-936558 (anti-PD-1, ONO-4538) plus Ipilimumab beim chemotherapienaiven } \\
\text { NSCLC (Erstlinie): Ergebnisse zu Ansprechen, Überleben }\end{array}$ \\
\hline Gettinger SN et al., 8024 & II & $\begin{array}{l}\text { BMS-936558 (anti-PD-1, ONO-4538) beim therapienaiven, fortgeschrittenen } \\
\text { NSCLC: Daten zu Sicherheit, Wirksamkeit, Korrelation des Baseline-PD-L1-Status } \\
\text { mit Outcome }\end{array}$ \\
\hline Rizvi NA et al., 8022 & I & $\begin{array}{l}\text { BMS-936558 (anti-PD-1, ONO-4538) plus Erlotinib beim EGFR-mutierten, fortge- } \\
\text { schrittenen (IIIB/IV) und chemotherapienaiven NSCLC: Daten zu Sicherheit und } \\
\text { Ansprechen }\end{array}$ \\
\hline $\begin{array}{l}\text { Carbone DP et al., TPS } 8128 \\
\text { (NCT02041533) }\end{array}$ & III & $\begin{array}{l}\text { BMS-936558 (anti-PD-1, ONO-4538) beim chemotherapienaiven NSCLC (IV) } \\
\text { oder NSCLC-Rezidiv mit PD-L1-positivem Tumor in Kombination mit Chemothe- } \\
\text { rapie nach Wahl des Studienarztes, offenes Studiendesign }\end{array}$ \\
\hline Rizvi NA et al., 8007 & I & $\begin{array}{l}\text { MK-3475 (anti-PD-1) beim fortgeschrittenen, systemisch nicht vorbehandelten } \\
\text { NSCLC (IV): Daten zu Sicherheit und klinischer Aktivität, Korrelation des Base- } \\
\text { line-PD-L1-Status mit Ansprechen (PD-L1-positiv } \geq 1 \% \text { ) }\end{array}$ \\
\hline Garon E et al., 8020 & I & $\begin{array}{l}\text { MK-3475 (anti-PD-1) beim vorbehandelten NSCLC: Daten zu Sicherheit und } \\
\text { klinischer Aktivität, Korrelation des Baseline-PD-L1-Status mit Ansprechen }\end{array}$ \\
\hline Brahmer JR et al., 8021 & I & $\begin{array}{l}\text { MEDI-4736 (anti-PD-L1) beim therapierefraktären, fortgeschrittenen NSCLC: } \\
\text { Daten zu klinischer Aktivität, Biomarker }\end{array}$ \\
\hline
\end{tabular}

präsentierten Phase-1b-Pilotstudie (CA209-003) mit einem gegen den PD-1-Rezeptor gerichteten Checkpoint-Inhibitor (anti-PD-1; Nivolumab) vor. Das Poster zeigte die Auswertung der 1- und 2-Jahres-Gesamtüberlebensdaten in der NSCLC-Kohorte $(\mathrm{n}=129)$, die dazugehörigen Sicherheitsund Subgruppenanalysen zur Tumorhistologie, aber auch Auswertungen zu potenziell prädiktiven Biomarkern wie der PD-L1-Expression, sofern ein Follow-up von $>1$ Jahr vorlag. Trotz des fortgeschrittenen Tumorstadiums und zahlreicher Vortherapien scheint ein Teil der Patienten langanhaltend auf die Therapie anzusprechen. Die Therapieeffekte ließen sich unabhängig vom histologischen Subtyp (Plattenepithel- und Nicht-Plattenepithelkarzinom) und unabhängig vom Mutationsstatus beobachten [12].

\section{Rationale für Kombinationsstrategien beim NSCLC}

Perspektivisch sind Checkpoint-Inhibitoren aufgrund ihrer Wirksamkeit, aber auch aufgrund des moderaten Toxizitätsprofils attraktive Kombinationspartner für zielgerichtete Therapien, Chemotherapie, Strahlentherapie, aber auch andere Immuntherapeutika. Dabei scheint eine empirische Herangehensweise (Kombination von 2 unterschiedlich wirksamen Therapieprinzipien) ebenso denkbar wie die Erarbeitung biologisch rationaler, auf dem molekularen Verständnis beruhender Ansätze. Für letztere gibt es zwar bereits interessante prä- klinische Ansätze, letztlich sind jedoch noch weitere Erkenntnisse zur Wirksamkeit von anti-PD1-/anti-PDL1-Inhibitoren in spezifischen Patientensubgruppen notwendig.

\section{Das Nierenzellkarzinom - \\ Tumor mit hohem immunogenem Potenzial}

Das Nierenzellkarzinom (renal cell cancer, RCC) repräsentiert mit einem Anteil von etwa $90 \%$ den häufigsten soliden Tumor der Niere [13]. Entscheidend für die Prognose der Patienten ist die Metastasierung: Bis zu 30\% der Patienten haben bei Diagnosestellung bereits Metastasen entwickelt, wobei das 5-Jahres-Überleben im fortgeschrittenen Stadium bei weniger als $10 \%$ liegt [14]. Die Etablierung molekular zielgerichteter sowie antiangiogener Substanzen führte zwar zu einer Verbesserung der Therapiemöglichkeiten und Überlebensraten [15], doch erlangt nur ein geringer Teil der Patienten eine vollständige Remission. In den meisten Fällen kommt es im Krankheitsverlauf zu einer Resistenzentwicklung und zur erneuten Progression: Die Mehrheit der Patienten verstirbt nach wie vor an den Folgen der Tumorerkrankung.

Ähnlich wie das maligne Melanom wird auch das RCC bereits seit Längerem als Tumor mit immunogenem Potenzial betrachtet und deshalb für klinische Versuche herangezogen [16]. Für die potenzielle Eignung immunbasierter Therapie-an- 
Abb. 1. Design der Phase-1b-Studie zur Prüfung eines PD-1-Antikörpers als Monotherapie bei verschiedenen soliden Tumoren (modifiziert nach [18]).

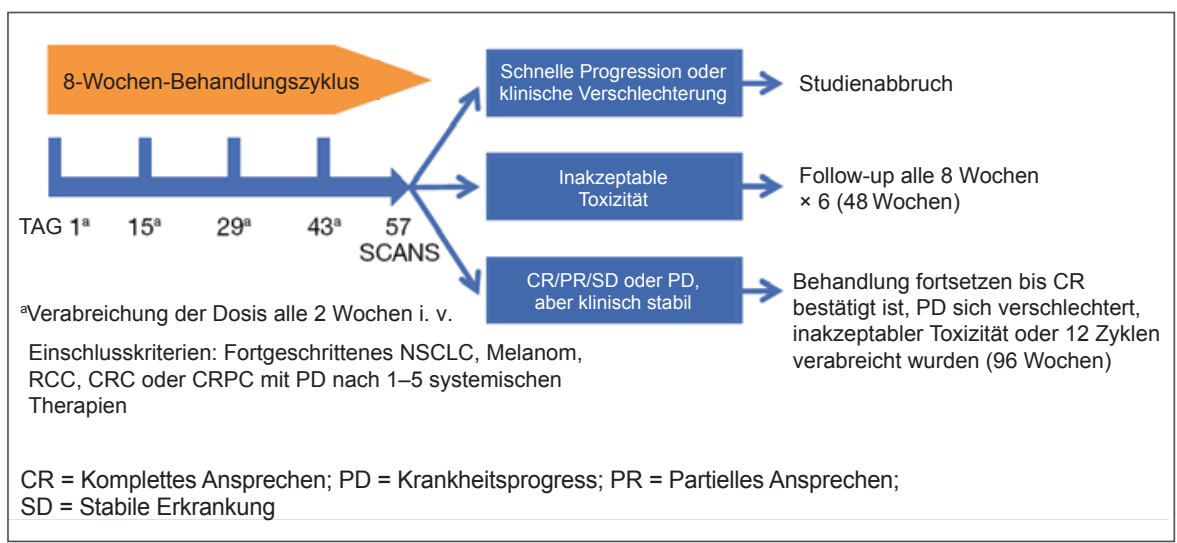

sätze sprechen auch hier kasuistisch berichtete Fälle von Spontanremissionen, die sich beim fortgeschrittenen RCC ereignen, sowie die auf Immunreaktionen hinweisende Infiltration des Tumorgewebes mit tumorspezifischen Immunzellen.

\section{Neue Therapiepotenziale durch Immun-Checkpoint-Inhibitoren}

Erste klinische Studien haben unter anderem zeigen können, dass die Inhibition des PD-1/PD-L1-Signalwegs auch beim therapierefraktären RCC ein Ansprechen des Tumors induzieren kann $[9,17,18]$. Aufgrund erster ermutigender klinischer Daten gehörten die neuen Studiendaten beim fortgeschrittenen RCC zu den meist diskutierten «Hot Topics» auf der diesjährigen Jahrestagung der ASCO [19, 20]. Bereits 2012 publizierten Topalian et al. [8] vielversprechende Daten, die auf das indikationsübergreifend große Potenzial des gegen PD-1 gerichteten Therapieansatzes hinwiesen (Abb. 1). Man hofft, mit den Immun-Checkpoint-Modifiern eine wertvolle Erweiterung des therapeutischen Armamentariums beim RCC gefunden zu haben.

\section{Neue ASCO-Daten zur Anti-PD-1/PD-L1-Blockade beim} fortgeschrittenen RCC

Die Arbeitsgruppe von Motzer et al. [21] stellte eine randomisierte dreiarmige Phase-II-Studie mit dem neuen gegen PD-1-gerichteten Immun-Checkpoint-Inhibitor Nivolumab bei Patienten mit therapierefraktärem, vorbehandeltem, meta- stasiertem RCC vor (metastatic RCC, mRCC). Die Patienten konnten im Vorfeld der CheckMate-016-Studie bis zu 3 systemische Therapielinien erhalten haben. Basierend auf den ersten Ergebnissen dieser Studie wurde ein Phase-III-Studienprogramm aufgesetzt. Bislang bietet die CheckMate-016-Studie die derzeit größte Datensammlung, die zur therapeutischen PD-1-Blockade beim mRCC verfügbar ist [19].

\section{Kombinationsstrategien mit dem \\ PD-1-Checkpoint-Inhibitor-Therapieprinzip}

Das Wirkprofil der Immun-Checkpoint-Inhibitoren lässt sie künftig auch als «Backbone-Therapie» im Rahmen von Kombinationsstrategien beim mRCC infrage kommen: Im günstigsten Fall könnten kombinierte Therapieansätze die Remissionsqualität weiter verbessern und zusätzliche Vorteile beim Gesamtüberleben generieren. Erste Phase-I-Studien, die in dieser Fragstellung während der diesjährigen ASCO-Tagung vorgestellt wurden, sprechen für eine Weiterentwicklung der neuen Therapieansätze in Phase-II/III-Studien [19, 20]. Eine der Limitierungen der Therapie ist allerdings die begleitende Toxizität, sodass diese Kombination weiterhin die Frage nach dem optimalen Kombinationspartner und dem besten Patientenkollektiv offen lässt.

\section{Disclosure Statement}

Die Autoren waren Referenten beim «4. Interdisziplinären Expertenforum», das von Bristol-Myers Squibb unterstützt wurde.

\section{Literatur}

1 Griesinger F, Eberhardt W, Früh M, et al.: DGHOLeitlinie «Lungenkarzinom, nicht-kleinzellig (NSCLC)». Stand: Oktober 2012. www.dgho.de.

2 MacConaill LE: Advancing personalized cancer medicine in lung cancer. Arch Pathol Lab Med 2012;136:1210-1216
Molina JR, Yang P, Cassivi SD, et al.: Non-small cell lung cancer: epidemiology, risk factors, treatment, and survivorship. Mayo Clin Proc 2008;83: 584-594.

4 Schiller JH, Harrington D, Belani CP, et al.: Comparison of four chemotherapy regimens for advanced non-small-cell lung cancer. N Engl J Med 2002;346:92-98.
Mok TS, Wu YL, Thongprasert S, et al.: Gefitinib or carboplatin-paclitaxel in pulmonary adenocarcinoma. N Engl J Med 2009;361:947-957.

6 Rosell R, Moran T, Oueralt C, et al.: Screening for epidermal growth factor receptor mutations in lung cancer. N Engl J Med 2009;361:958-967.

7 Kwak EL, Bang YJ, Camidge DR, et al.: Anaplastic lymphoma kinase inhibition in non-small-cell lung cancer. N Engl J Med 2010;363:1693-1703. 
8 Topalian SL, Hodi FS, Brahmer JR, et al.: Safety, activity, and immune correlates of anti-PD-1 antibody in cancer. N Engl J Med 2012;366:2443-2454.

9 Brahmer JR, Tykodi SS, Chow LQ, et al.: Safety and activity of anti-PD-L1 antibody in patients with advanced cancer. N Engl J Med 2012;366: 2455-2465.

10 Lawrence MS, Stojanov P, Polak P, et al.: Mutational heterogeneity in cancer and the search for new cancer-associated genes. Nature 2013;499: 214-218.

11 Topalian SL, Weiner GJ, Pardoll DM: Cancer immunotherapy comes of age. J Clin Oncol 2011; 29:4828-4836.

12 Brahmer JR, Horn L, Gandhi L, et al.: Nivolumab (anti-PD-1, BMS-936558, ONO-4538) in patients (pts) with advanced non-small-cell lung cancer (NSCLC): Survival and clinical activity by subgroup analysis. J Clin Oncol 2014;32:5s(suppl):abstr 8112.
13 Kirchner H, Casper J, Gauler T: DGHO-Leitlinie «Nierenzellkarzinom (Hypernephrom)». Stand Februar 2013.www.dgho.de.

14 Van Poppel H, Ameye F: Chemotherapeutic agents for urologic oncology; in Chapple CR, Steers WD (eds): Practical Urology: Essential Principles and Practice. London, Springer, 2011.

15 Hutson TE: Targeted therapies for the treatment of metastatic renal cell carcinoma: clinical evidence. Oncologist 2011;16(suppl 2):14-22.

16 McDermott DF: Immunotherapy of metastatic renal cell carcinoma. Cancer 2009;115:2298-2305.

17 Herbst RS, Gordon MS, Fine GD, et al.: A study of MPDL3280A, an engineered PD-L1 antibody in patients with locally advanced or metastatic tumors. J Clin Oncol 2013;31(suppl):abstr 3000
18 Hodi FS, Topalian SL, Brahmer JR, et al.: Survival, efficacy, and long-term safety in patients with advanced solid tumors receiving nivolumab (antiPD-1; BMS-936558; ONO-4538). European Cancer Congress 2013; abstr 880.

19 Clinical Science Symposium: «Unleashing the Immune System in Genitourinary Cancers», 2014 ASCO Annual Meeting, 31.05.2014 (Abstracts \#5009, \#5010,\#5012).

20 Oral Abstract Session: «Genitourinary (Nonprostate) Cancer» 2014, ASCO Annual Meeting, 2.6.2014: (Abstract: \#4504).

21 Motzer RJ, Rini BI, McDermott DF, et al. Nivolumab for metastatic renal cell carcinoma (mRCC): results of a randomized, dose-ranging phase II trial. J Clin Oncol 2014;32:5s(suppl):abstr 5009). 